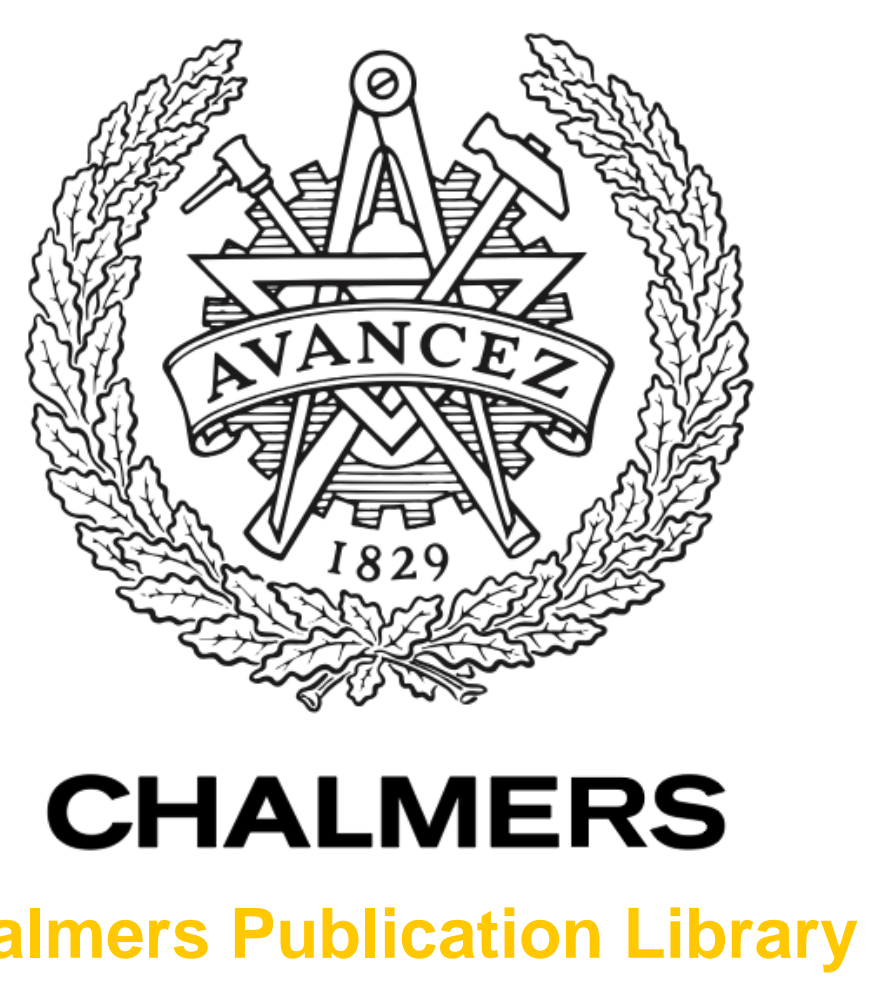

Chalmers Publication Library

\title{
A Receding Horizon Approach for Designing String Stable Cooperative Adaptive Cruise Control
}

This document has been downloaded from Chalmers Publication Library $(\mathrm{CPL})$. It is the author's version of a work that was accepted for publication in:

IEEE Conference on Intelligent Transportation Systems - ITSC 2011, October 5-7, 2011, Washington, DC, USA (ISSN: 2153-0009)

Citation for the published paper:

Kianfar, R. ; Falcone, P. ; Fredriksson, J. (2011) "A Receding Horizon Approach for Designing String Stable Cooperative Adaptive Cruise Control". IEEE Conference on Intelligent Transportation Systems - ITSC 2011, October 5-7, 2011, Washington, DC, USA pp. 734-739.

Downloaded from: http://publications.lib.chalmers.se/publication/143468

Notice: Changes introduced as a result of publishing processes such as copy-editing and formatting may not be reflected in this document. For a definitive version of this work, please refer to the published source. Please note that access to the published version might require a subscription. 


\title{
A Receding Horizon Approach to String Stable Cooperative Adaptive Cruise Control
}

\author{
Roozbeh Kianfar, Paolo Falcone, Jonas Fredriksson
}

\begin{abstract}
A time domain approach to a "string stable", i.e., capable of attenuating acceleration shockwaves, cooperative adaptive cruise control is proposed in this paper. $A$ receding horizon scheme is adopted to design a controller which attenuates acceleration shockwaves generated by the preceding vehicle while avoiding rear-end collisions. The classical definition of string stability in frequency domain is revised in the time domain and a new criterion for predecessor-follower string stability based on the acceleration signals is defined and used. Simulation and experimental results are presented to show the effectiveness of the proposed method.
\end{abstract}

\section{INTRODUCTION}

The increasing number of vehicle as well as the growth of road transportation can lead to traffic flow problems, like congestions, which cause time losses, affect the environmental load and increase the possibility of accidents. Intelligent Transportation Systems (ITS) can contribute to alleviate traffic problems like congestions. Cooperative driving is an example of an ITS technology, which consists of hardware and software mechanisms enabling autonomous driving of multiple vehicles (vehicle platoons) to achieve a common objective. Vehicles in a platoon cooperatively decide their behavior in order to achieve a common goal like, e.g., increasing road throughput, attenuating traffic shock-waves, reducing fuel consumption and pollutant emissions.

An efficient way to increase the road traffic throughput is to reduce the inter-vehicle distance. Unfortunately, driver's reaction time is subjected to delay, i.e., it takes some time until the driver reacts to changes in the environment. This delay can have a great impact on the traffic flow, which can as indicated earlier result in collisions or other undesired phenomena in traffic. In other words, reducing inter-vehicle distance conflicts with traffic safety. Automated driving can instead, help to safely reduce the inter-vehicle distance thus lead to increased traffic throughput. Adaptive Cruise Control (ACC) is a functionality, common in high-end modern vehicles, which automates the longitudinal control of the vehicles. Standard ACC functionality is designed to increase comfortability of the driver, while keeping a desired, short but safe distance to the preceding vehicle. In addition, ACC can reduce rear-end collisions and fuel consumption [5].

However, using standard ACC functionality to control the longitudinal motion of a vehicle platoon might not be suitable, due to string stability problems. String stability can

Roozbeh Kianfar, Paolo Falcone and Jonas Fredriksson are with Department of Signals and Systems, Mechatronics, Chalmers University of Technology, 41296 SE, Göteborg, Sweden roozbeh, falcone, jonas. fredriksson@chalmers.se be described as the ability of a platoon in attenuating the relative distance errors when it propagates towards the tail of the platoon, [8]. String instability causes oscillation or even unnecessary traffic jam in the platoon. String stability is defined for different type (homogenous and heterogeneous) and different setups, such as defined for leader-predecessorfollower strategy or predecessor-follower strategy, see e.g. [1], [6], [10]. It is known that string stability criterion for a predecessor-follower strategy is more conservative compared to a lead-predecessor-follower strategy, but it is more desirable from an implementation point of view since communication only with the preceding vehicle is required. Homogeneous platoon refer to a string of vehicles where all the vehicles in the string are identical, in contrast to heterogeneous platoon where the vehicles can be of different kind, from small passenger cars to long-haul transportation trucks with different arbitrary controllers.

In [8], [10], [11] string stability of homogeneous and heterogeneous platoons is extensively investigated in frequency domain. Unfortunately, including constraints, e.g., collisionavoidance constraint in frequency domain design is a difficult task. Model predictive control is a powerful time domain tool to satisfy multiple constraints. However, interpretation of the state of the art results in frequency domain, e.g., string stability condition, in time domain is not trivial.

In [6], it is shown that the inter-vehicle distance, hence the traffic throughput, can be minimized further by using Cooperative Adaptive Cruise Control (CACC). CACC is an extended version of ACC, which utilizes wireless communication between vehicles to exchange information about the vehicle current state and future intention.

In this paper, a decentralized receding horizon approach is used to design a set of local controllers enforcing string stability while guaranteeing collision avoidance. Hence, a time domain interpretation of string stability is proposed which let us treat string stability as a constraint in the optimization problem. To guarantee collision avoidance, safety constraints are added to the optimization problem. To assess the effectiveness of the method both simulation and experiment are carried out.

The paper is organized as follows. In section 2, intervehicle dynamics of a platoon is derived. In the third section the control problem is described in details. In section 4 , a model predictive scheme is used to design the controller, which satisfies multiple constraints. Simulation and experimental results are presented in section 5 and 6 . Finally in section 7 , the conclusions are drawn. 


\section{MODELING OF INTER-VEHICLE DYNAMICS}

In CACC design, it is interesting to study the inter-vehicle dynamics, such as relative position and relative velocity between two or several vehicles. Consider two adjacent vehicles in Fig. 1, let $p_{i}, v_{i}$ and $a_{i}$ denote the position, velocity and acceleration of the preceding vehicle and $p_{i+1}, v_{i+1}$ and $a_{i+1}$ denote the position, velocity and acceleration of the following vehicle (hereafter also called the ego vehicle) in a platoon, respectively. Denote by $e_{p}$, as the position

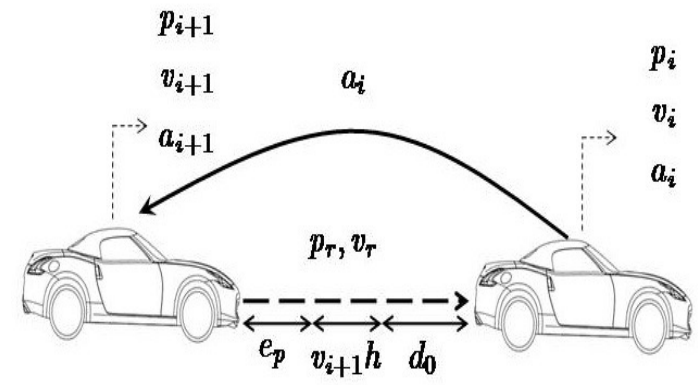

Fig. 1. Two adjacent vehicles in the platoon

error between the desired distance with respect to preceding vehicle, i.e., $e_{p}=p_{i}-p_{i+1}-d_{0}-v_{i+1} h_{i+1}$, where $d_{0}$ and $h_{i+1}$ are the constant safety distance and the headway time, respectively. The headway time is the time that a vehicle takes to reach the preceding vehicle while traveling at its current speed. Also, let $e_{v}$, be the relative velocity between the two vehicles, i.e., $e_{v}=v_{i}-v_{i+1}$. Since, we are interested in the inter-vehicle dynamics, we differentiate the errors with respect to time, the error dynamics are then described by the following set of equations:

$$
\begin{aligned}
& \dot{e}_{p}=e_{v}-a_{i+1} h_{i+1}, \\
& \dot{e}_{v}=a_{i}-a_{i+1},
\end{aligned}
$$

The acceleration of the ego vehicle, $a_{i+1}$ is assumed to be described by the following simplified model as follows,

$$
a_{i+1}=\frac{K_{i+1}}{\tau_{i+1} s+1} u_{i+1},
$$

where $K_{i+1}$ and $\tau_{i+1}$ are the steady state gain and the time constant of the actuator (engine and brake), respectively and $u_{i+1}$ is the demanded acceleration. This model is often proposed in the literature, see e.g. [7]. The model (1)-(2) of two adjacent vehicles in a platoon can be written in the state-space form as

$$
\dot{x}=A x+B_{1} u+B_{2} \nu
$$

where

$$
\begin{aligned}
A= & {\left[\begin{array}{cccc}
0 & 1 & -h_{i+1} & 0 \\
0 & 0 & -1 & 0 \\
0 & 0 & -1 / \tau_{i+1} & 0 \\
0 & 0 & 1 & 0
\end{array}\right], } \\
B_{1}= & {\left[\begin{array}{c}
0 \\
0 \\
\frac{K_{i+1}}{\tau_{i+1}} \\
0
\end{array}\right], B_{2}=\left[\begin{array}{l}
0 \\
1 \\
0 \\
0
\end{array}\right], } \\
x= & {\left[\begin{array}{cccc}
e_{p} & e_{v} & a_{i+1} & v_{i+1}
\end{array}\right]^{\mathrm{T}}, }
\end{aligned}
$$

where $x, u$ and $\nu$ are the state vector, the control signal and disturbance, respectively.

String stability is an important property of a platoon, which refers to the capability of the vehicles in the platoon in attenuating traffic shockwaves and is defined next.

String stability. In general, string stability of a platoon is defined as the platoon capability in attenuating the spacing errors upstream in the string. It means that the spacing error between vehicles should not be amplified when it propagate towards the tail of the platoon, see e.g., [4], [10], i.e., if $e_{i}$ and $e_{i+1}$ are the spacing error between two adjacent vehicles, then to guarantee string stability the following condition should be satisfied:

$$
G(s)=\left\|\frac{e_{i-1}}{e_{i}}\right\|_{\infty} \leq 1
$$

where $\mathrm{G}(\mathrm{s})$ is the transfer function. This definition is adopted by many researchers such as [8], [9], [10]. However, in this work, the following condition

$$
\left\|\frac{a_{i}}{a_{i+1}}\right\|_{\infty} \leq 1
$$

is used to guarantee that the acceleration of the preceding vehicle is not amplified by other vehicles upstream in the platoon. In (8), with a slight abuse of notation for the sake of readability $a_{i}(s)$ and $a_{i+1}(s)$ are the acceleration of two adjacent vehicles in Laplace domain. This condition, (8), is used in Grand Cooperative Driving Challenge (GCDC) competition [3], and is adopted in this work. The main goal is to develop a controller that can minimize the position and velocity errors and guarantee safety while enforcing string stability condition in a platoon.

\section{PROBLEM STATEMENT}

The control objective is to minimize the position and velocity errors subjected to a number of constraints which will be described next. The constraints are resulting from different requirements, such as safety, performance, drivability etc. The specifications are described below and the related constraints are formalized:

- Safety: Safety or collision avoidance constraints are required to guarantee that the controller always keep a safe distance from the preceding vehicle to avoid collisions. This constraint can be written as

$$
0 \leq e_{p}(t) \leq e_{p, \max }, \quad \forall t \geq 0,
$$


where $e_{p, \max }$ is the maximum allowed distance from the desired distance. It should be noted that, violation of lower bound in (9) means that the ego vehicle has a shorter distance to the preceding vehicle than the safe distance.

- Performance: In standard ACC controller, the velocity of preceding vehicle has to be tracked. Hence, in order to avoid large deviation from the desired speed, the relative speed between the two adjacent vehicles is also constrained, i.e.,

$$
e_{v, \min } \leq e_{v}(t) \leq e_{v, \max }, \quad \forall t \geq 0
$$

This constraint also enforces the vehicle to operate within a desired speed range.

- Actuator limitations: To ensure that the acceleration commanded by the controller is within the admissible actuator range, the following constraint is introduced

$$
u_{\min } \leq u(t) \leq u_{\max }, \quad \forall t \geq 0,
$$

The limits, $u_{\min }$ and $u_{\max }$, can be global limits for all vehicles in a platoon.

- Drivability and fuel consumption: To preserve comfort for the driver and the passengers and improving the fuel consumption, the rate of control signal is limited as follows:

$$
\dot{u}_{\min } \leq \dot{u}(t) \leq \dot{u}_{\max }, \quad \forall t \geq 0,
$$

\section{PROBLEM FORMULATION}

In this section, we design local controllers according to a receding horizon control framework. We recall that the control objective of each vehicle is to regulate to zero the position and velocity errors $e_{p}$ and $e_{v}$, respectively, while satisfying the constraints described in Section III.

We assume that the state and the disturbance vectors can be measured every sampling time instant $t_{s}$, and solve the following optimization problem in receding horizon

$$
\begin{aligned}
& \min _{\delta U_{t}, \varepsilon} \sum_{k=0}^{N-1}\|y(t+k \mid t)\|_{Q}^{2}+\|\delta u(t+k \mid t)\|_{R}^{2} \\
& +\|u(t+k \mid t)\|_{W}^{2}+\rho \varepsilon^{2}
\end{aligned}
$$

\section{subject to:}

$$
\begin{aligned}
x(t+k+1 \mid t) & =F x(t+k \mid t)+G_{1} u(t+k \mid t) \\
& +G_{2} \nu(t+k \mid t) \\
y(t+k+1 \mid t) & =H x(t+k \mid t) \\
u(t+k \mid t) & =u(t+k-1 \mid t)+\delta u(t+k) \\
\nu(t+k \mid t) & =\nu(t \mid t) \\
& k=0, \ldots, N-1 \\
u(t-1 \mid t)= & u(t-1) \\
\nu(t \mid t)= & \nu(t) \\
x(t \mid t) & =x(t) \\
x_{\min }(t)-\varepsilon \leq & x(t+k \mid t) \leq x_{\max }(t)+\varepsilon \\
u_{\min } \leq & u(t+k \mid t) \leq u_{\max } \\
\delta u_{\min } \leq & \delta u(t+k \mid t) \leq \delta u_{\max } \\
\varepsilon & \geq 0,
\end{aligned}
$$

where $\delta U_{t}=[\delta u(t), \ldots, \delta u(t+N-1)]$ is the vector of future input increments, i.e., the vector of optimization variables, $N$ is the prediction horizon length, $\varepsilon$ is a slack variable introduced to soften the constraints (21), $Q \succeq 0, R \succ 0$ and $W \succeq 0$ are weighting matrices of appropriate dimensions and $\rho>0$ penalizes the slack variable and $y=\left[e_{p}, e_{v}\right]^{T}$ is the output vector. The matrices $F, G_{1}$ and $G_{2}$ are obtained by discretizing the system (3) with a sampling time $t_{s}$. Constraints (21) include the safety and the performance constraints (9)-(10), respectively, introduced in Section III, while constraints (22) account for actuators limitations. Finally, constraints (23) guarantee passengers comfort.

Next we discuss how to modify the problem (13)-(24) in order to enforce string stability.

The string stability criterion (8) is defined in the frequency domain. However, for the distributed MPC scheme considered in this paper, a time domain criterion is needed. A straightforward definition of string stability in the time domain follows.

Definition 1 (String stability): A vehicle platoon is string stable if for a step change in the velocity of the leader vehicle $v_{1}(t)$ at time $t=0$, there exist constant scalars $\gamma_{i} \in(0,1), i=2, \ldots, N$ such that,

$$
\max _{t \geq 0}\left|a_{i+1}(t)\right| \leq \gamma_{i} \max _{t \geq 0}\left|a_{i}(t)\right|, i=2, \ldots, N-1 .
$$

The condition (25) states that, in a speed change maneuver of the leader, the acceleration response of each vehicle in a string stable platoon should not exceed the acceleration of the preceding vehicle. Local controllers have then to be designed such that the (25) holds. In the distributed receding horizon framework considered in this paper, additional constraints are included in the local controller to enforce (25). Formulating general local constraints rigorously guaranteeing the (25) is not trivial. In this paper we present a practical way to enforce the attenuation of the acceleration signals.

We observe that in a speed change, each vehicle in the platoon should mimic the behavior of the preceding with a 
delay. Hence, the following constraints can be added to the problem (13)-(23)

$a_{i+1}\left(t \mid t_{k}\right) \leq \gamma \max _{\tau \in\left[t_{k}-H, t_{k}\right]}\left|a_{i}(\tau)\right| \quad$ for $t \in\left[t_{k}, t_{k}+N\right]$

where $t_{k}$, is the time instant when the optimal control signal is calculated, $N$ is the prediction horizon length and $H$ is the size of time window, which is a tuning parameter. The condition (26) means that at every time instant $t_{k}$ the acceleration of the ego vehicle is bounded by the acceleration profile of the preceding, over a past time interval of length $H$. Hence, the parameter $H$ must be chosen long enough in order to account for the delay arising from different dynamics within the platoon.

\section{SIMULATION RESULTS}

In this section we present simulation results where, for the sake of simplicity, only two vehicles are considered. The preceding (leader) vehicle is described by a double integrator, fed with acceleration measurements logged in test experiments and shown in Fig. 2 with a solid line.

In order to verify the capability of the proposed controller of attenuating the acceleration signal of the following vehicle, a maneuver is chosen, where the leading vehicle starts from the standing still position and then, after acceleration and deceleration maneuvers ends by hard braking and reducing the velocity down to standing still again. Simulations are performed by using the Model predictive Control Toolbox in Matlab and Simulink [2]. The time constant $\tau$ and steady state gain $K$ in the prediction model (2) are identified based on experimental data logged in a Volvo S60 as $\tau=0.4$ and $K=1$. The position error $e_{p}$ is defined by using a headway time $h$ and a constant safety distance $d_{0}$ of $1 \mathrm{sec}$ and $10 \mathrm{~m}$, respectively. The weighting matrices in the cost function (13) are $Q=\operatorname{diag}([6,8,3,0]), R=.8$ and $W=1$. Velocity and acceleration of the vehicles are constrained as follows:

$$
\begin{aligned}
0 & \leq v_{i+1} \leq 80 \quad\left[\frac{k m}{h}\right], \\
-4.5 & \leq a_{i+1} \leq 2.5 \quad\left[\frac{m}{s^{2}}\right] . \\
-4.5 & \leq u_{i+1} \leq 2.5 \quad\left[\frac{m}{s^{2}}\right] . \\
-3 & \leq \dot{u}_{i+1} \leq 3 \quad\left[\frac{m}{s^{3}}\right] .
\end{aligned}
$$

Fig. 2, shows the acceleration profiles of ego and preceding vehicles. We observe that the acceleration of the preceding vehicle is not amplified by the ego vehicle, that is, the string stability criterion (8) is satisfied. Fig. 3, shows the position error from the desired distance. Clearly, the error should be as small as possible, thus resulting in a shorter platoon, yet avoiding negative error. However, it can be seen that around $t=48 \mathrm{sec}$, to have a feasible solution, negative position error is unavoidable. In Fig. 4, the velocity profiles of preceding and ego vehicles are presented which indicates a good speed tracking and finally, Fig. 5 shows the commanded acceleration from the controller to the vehicle.

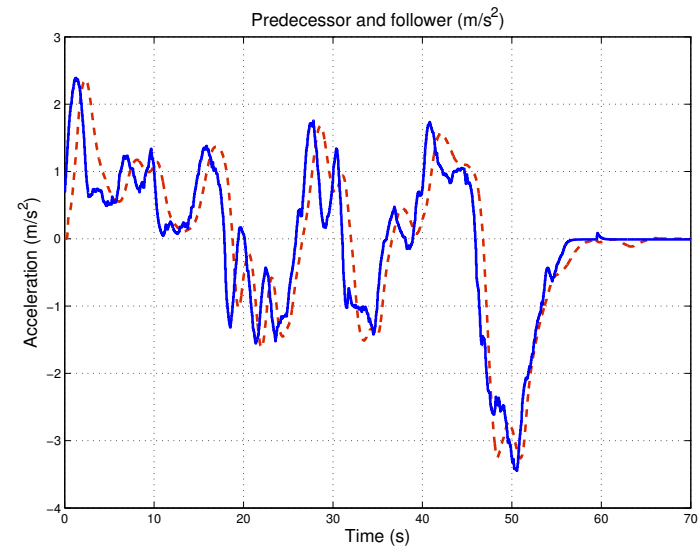

Fig. 2. Solid blue curve represents acceleration of preceding vehicle and dashed red curves shows the acceleration of ego vehicle.

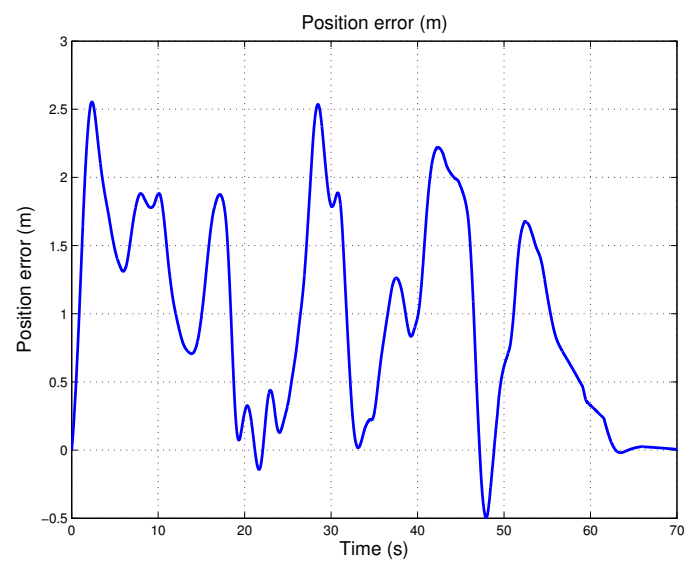

Fig. 3. Position error with respect to desired distance.

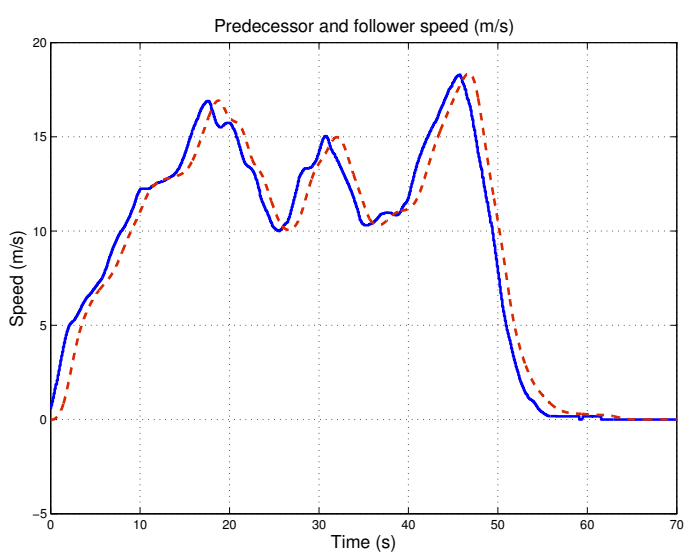

Fig. 4. Solid blue curve represents velocity of preceding vehicle and dashed red curves shows the velocity of ego vehicle.

\section{EXPERIMENTAL RESULTS}

The receding horizon controller presented in this paper has also been evaluated through experiments. where two vehicles are used. The lead vehicle is a Volvo S80 and the ego vehicle is a Volvo S60. The controller is executed in real 


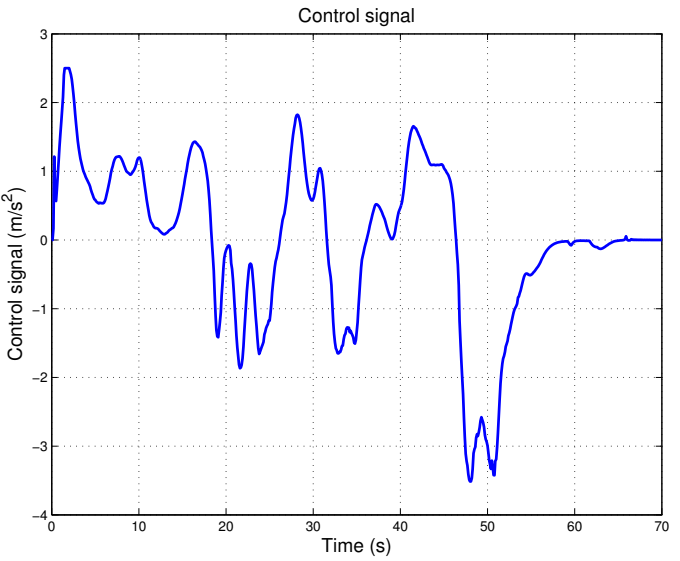

Fig. 5. Control signal.

time implementation on a dSpace MicroAutoBox platform. In Fig. 6, the ego vehicle and experimental setup in the trunk of S60 are shown.
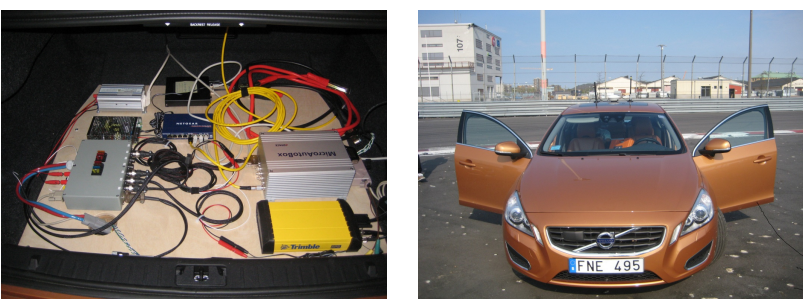

Fig. 6. Figure to the left shows the experimental setup in the trunk which are dSpace Autobox, GPS and so on. Figure to the right, is the Volvo S60 which is used for the test.

The ego vehicle is equipped with a radar, which measures the relative distance and velocity with respect to the preceding vehicle. The preceding vehicle sends its acceleration via wireless communication. The vehicle states are estimated through a Kalman filter. The control command is sent to the vehicle engine and brake units via CAN bus.

The experimental test is performed on a straight with the headway time set to $h=0.8 \mathrm{sec}$ and a safety distance of $d=$ $10 \mathrm{~m}$. The experiment starts with both vehicles at standing still. After performing few acceleration and deceleration cycles, both vehicles decelerates down to standing still.

In Fig. 7, experimental results are presented. As, it can be seen from the position error figure, in the beginning there is an $8 m$ error from the desired position. However, as the maneuver starts, the position error decreases until the end of maneuver when the ego vehicle stops almost exactly at the desired position. We recall that, the control objective is having zero position error, which results in the shortest allowed platoon length, while avoiding undershoot, which means that the vehicle crossed the minimum safety distance. This tradeoff makes the controller design more challenging. The experimental results show that the controller succeeds in maintaining the ego vehicle at the minimum allowed distance while avoiding the violation of the safety constraints.

The top figure, shows the commanded acceleration from
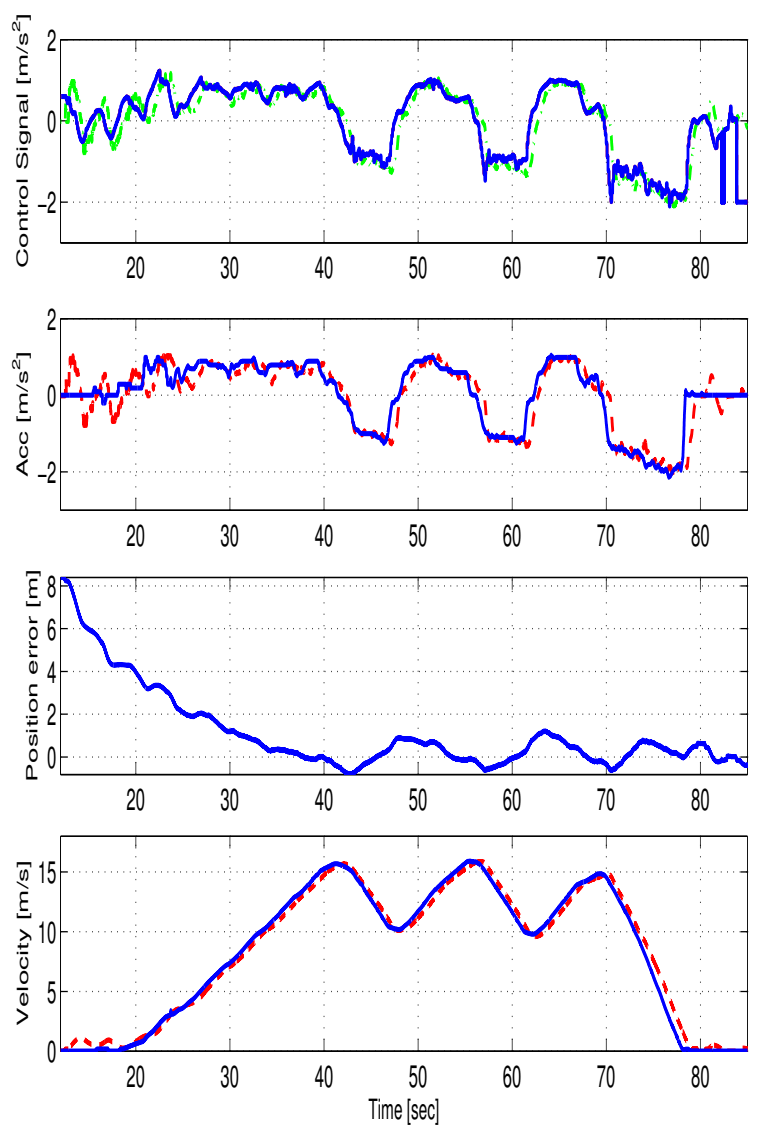

Fig. 7. In the top figure, the blue solid curve represents the commanded acceleration and the green dashed-dotted curve is vehicle acceleration. In the second figure, red dashed curve and blue curve are the acceleration of ego and preceding vehicle respectively. Third figure represents the position error. In the bottom figure, velocity profile of ego and preceding vehicle are shown in dashed red and blue respectively.

the controller and the vehicle acceleration. The second plot, represents the acceleration profile of ego and preceding vehicle. This plot, is particularly important to investigate string stability. As it can be seen from time domain response the controller almost never amplify the acceleration of preceding vehicle. The figure to the bottom shows the velocity profile of preceding and ego vehicle. We should note that more experimental tests are carried out which emphasize on the consistency of the controller performance. However, for the sake of presentation only one set of results is presented.

For further investigation about string stability, an empirical transfer function from acceleration of preceding vehicle (input) to acceleration of ego vehicle (output) is identified using system identification tools. The identified transfer function is a second order ARX model.

In Fig. 8, the frequency response of identified transfer function is plotted. The frequency response confirms the predecessor-following string stability of the platoon. 

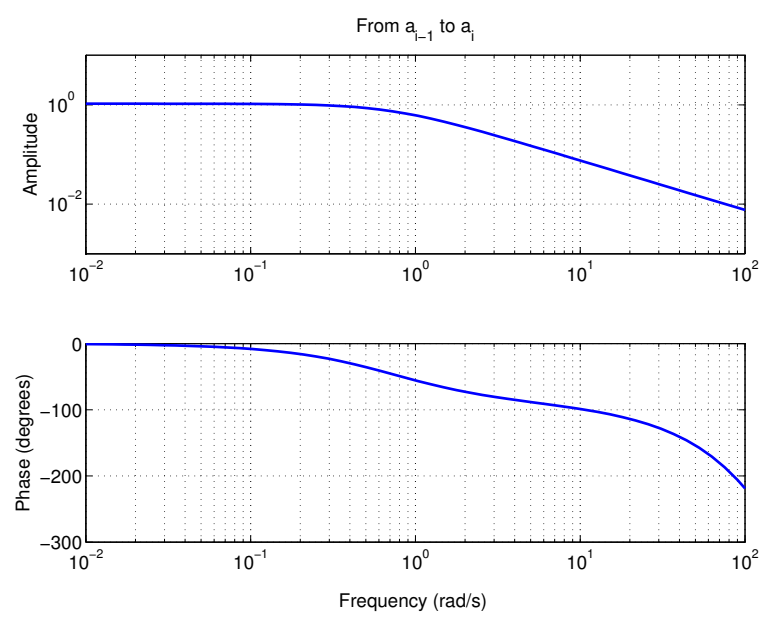

Fig. 8. Magnitude and phase of frequency response of identified transfer function.

\section{CONCLUSION}

In this paper, a receding horizon control approach is used to design a cooperative adaptive cruise control. Safety and string stability are enforced by imposing additional constraints to the optimization problem. Acceleration of preceding vehicle is used as a measured disturbance in the controller design, which results in faster response and shorter inter-vehicle distance. Simulation and experimental results show that the controller performs a good tracking while fulfilling the string stability and safety constraints. However, finding a systematic approach to tune the tuning parameter $H$ is a challenging task, which can be investigated further in future. Also, as the future work, the intent acceleration profile of preceding vehicle can also be used to improve the prediction model. Sensitivity analysis can be carried out to investigate the sensitivity of the controller with respect to communication delay and packet drops. Furthermore, simulation and experiment with more vehicles in a heterogeneous platoon can be carried out.

\section{ACKNOWLEDGMENTS}

The authors gratefully acknowledge Volvo Car Corporation and the Chalmers GCDC team for their support and willingness to carry out experiments.

\section{REFERENCES}

[1] P. Barooah and J. Hespanha. Error amplification and disturbance propagation in vehicle strings with decentralized linear control. In 44th IEEE Conference on Decision and Control, Boston, Massachusetts, USA, December 2005.

[2] A. Bemporad, M. Morari, and N. Lawrence Ricker. User Guide's for Model Predictive Control Toolbox in Matlab, 2010.

[3] http://www.gcdc.net. Grand Cooperative Driving Challenge. Helmond, Netherland, 2011.

[4] M. E. Khatir and E. J. Davison. Decentralized control of a large platoon of vehicles using non-identical controllers. In American Control Conference, Boston, Massachusetts, USA, 2004.

[5] F. Lattemann, K. Neiss, S. Terwen, and T. Connolly. The predictive cruise control a system to reduce fuel consumption of heavy duty trucks. In SAE, Rosemont, IL, USA, October 2004.
[6] G.J.L. Naus, Rene P.A . Vugts, J. Ploeg, J.G de Molengraft, and M. Steinbuch. String-stable CACC design and experimental validation : A frequency-domain approach. IEEE Transaction on vehicular technology, 59(9):4268-4279, 2010.

[7] R. Rajamani. Vehicle Dynamics and Control. Springer, 2005.

[8] P. Seiler, A. Pant, and K. Hedrick. Disturbance propagation in vehicle strings. IEEE TRANSACTIONS ON AUTOMATIC CONTROL, 49(10):1835-1841, 2004.

[9] Elaine Shaw and J. Karl Hedrick. Controller design for string stable heterogeneous vehicle strings. 2007 46th IEEE Conference on Decision and Control, pages 2868-2875, 2007.

[10] Elaine Shaw and J. Karl. Hedrick. String stability analysis for heterogeneous vehicle strings. 2007 American Control Conference, pages 3118-3125, July 2007.

[11] D. Swaroop and K. Hedrick. String stability of interconnected sysyems. IEEE TRANSACTIONS ON AUTOMATIC CONTROL, 41:349-357, 1996. 